\title{
"CONCLLUSIONI STORICHE. NUOVE RICERCHE SUL LIBRO DELLA SCALA E LA CONOSCENZA DELL'ISLAM IN OCCIDENTE»
}

Presentación, por

MÍKEL DE EPALZA

El profesor italiano Enrico Cerulli ha muerto, en 1988.

Con su muerte desaparece una gran figura de los estudios sobre literatura etiópica, pero sobre todo un especialista en las múltiples relaciones medievales entre la literatura árabe-islámica y las literaturas cristianas de Oriente y de Occidente. Embajador y hombre político, presidió en estas últimas décadas la Accademia Nazionali dei Lincei, la institución italiana que, desde el siglo XVII, aglutina a todas las Academias científicas de la península e islas adyacentes. Desde ese cargo le debemos agradecer - la cultura hispánica y particularmente la catalana y este presentador - su interés en publicar en sus colecciones la edición, traducción castellana y estudio de la obra árabe de Turmeda (La Tuhfa, autobiografía y polémica islámica contra el Cristianismo de 'Abdallāh al-Tarŷān (fray Anselmo Turmeda), Atti della Accademia Nazionale dei Lincei, anno CCCLXVIII - 19871).

Además de un pequeño estudio sobre el Patrañuelo, las letras árabes e hispánicas le deben unos estudios decisivos sobre la temática del Dante y sus antecedentes islámicos. Ya en 1949 publicaba su monumental El "Libro della Scala» e la questione delle fonti arabo-spagnole della Divina Commedia, cuyas conclusiones iba a desarrollar en una luminosa ponencia en 1956 ("Dante e I'Islam", Convegno... Oriente e Occidente nel Medio Evo, Roma, Accademia Nazionale dei Lincei, 1957, 275-294; reimpreso en la revista Al-Andalus, de Madrid). Finalmente, en 1972, iba a dar el broche final de estos estudios en Nuove ricerche sul Libro della Scala e la conoscenza dell'Islam in Occidente (Vaticano, 1972), cuyas conclusiones publicamos en nuestra revista, como homenaje al gran sabio desaparecido. 
La nostra ricerca può dirsi qui conclusa. Giova perciò fermarsi a considerarne brevemente $\mathrm{i}$ risultati, tenendo presenti questo volume e l'altro mio precedente (1).

1. La conoscenza della narrazione della Visione Oltretomba di Maometto è così da delimitare nelle letterature dell'Occidente medievale: Nella Spagna un singolo episodio è già citato nel codice di Uncastillo (poco dopo il 1222) ed un breve riassunto è nella Historia Arabum di Rodrigo Ximénez de Rada (anteriormente al 1247) donde è passato nella Crónica General del Re Alfonso $\mathrm{X}$ il Savio (anteriormente al 1284).

'Poi il testo completo della Visione di Maometto (Kitāb al-Mírǟg) fu fatto tradurre, per ordine del Re Alfonso X il Savio, dall'arabo in castigliano ad opera del medico della Corte di Castiglia: Abraham Alfaquím. Di tale traduzione, fatta tra il 1260 ed il 1264, non ci resta che un riassunto, in un codice dell'Escuriale, riassunto inserito in un'opera attribuita a S. Pedro Pascual. Ma lo stesso Alfonso il Savio aveva poi fatto ancora tradurre dal castigliano in francese ed in latino quel racconto della Visione Oltretomba; ed il traduttore fu l'esule toscano ghibellino Bonaventura da Siena. Della traduzione, che a rendere l'arabo Kitāb al-Mir räğ si ebbe il titolo di 'Libro della Scala', abbiamo ora il testo francese completo in un codice della Bodleiana di Oxford, datato dal maggio 1264; ed il testo latino completo in un codice della Bibliothèque Nationale di Parigi, copiato in Brettagna nel secolo XIV ed un codice della Biblioteca Vaticana, anche esso del primo trentennio del secolo XIV.

1 testi francese e latino ed il riassunto del testo castigliano furono da me editi nel primo volume di quest'opera.

2. La narrazione della Visione Oltretomba nel Libro della Scala è presentata come fatta dallo stesso Maometto in prima persona; e perciò si credette in Occidente che esso fosse uno dei libri sacri dell'Islam, tanto che in alcuni codici venne inserito, e quindi tra-

(1) E. Cerulli, Il Libro della Scala e la questione delle fonti arabo-spagnole della Divina Commeãia, Città del Vaticano 1949. 
smesso, nella Collectio Toletana delle opere, dal Corano in poi, ritenute in Occidente come sacre per i Musulmani e già fatte tradurre dall'arabo in latino nel 1141 da Pietro il Venerabile, abbate di Cluny, durante la sua missione a Toledo. In realtà invece il Libro della Scala non è affatto un libro sacro dell'Islam, ma soltanto un'opera di pietà popolare. Però esso venne diffuso nell'Europa a datare dalla metà del secolo XIII come la Visione, sacra per i Musulmani, di un viaggio al Paradiso ed all'Inferno.

3. Quali testimonianze della diffusione del Libro della Scala risultano da queste mie ricerche?

a) Passi del Libro della Scala sono citati: dallo stesso Re Alfonso il Savio nel suo Setenario, dunque prima del 1284; da Fazio degli Uberti nel suo Dittamondo (tra il 1350 ed il 1360); da Francesco Eiximenis nel Libre del Crestiá (alla fine del Trecento e comunque anteriormente al 1408); da Antonio Torquemada (prima del 1549) e dal suo traduttore Celio Malaspini (prima del 1590).

b) Riassunto diffuso e particolareggiato di tutto il Libro della Scala in latino nel Fortalitium Fidei di Alfonso da Spina (anteriormente al 1485); lungo riassunto in italiano di Roberto Caracciolo da Lecce (anteriormente al 1495); ancora un lungo, totale riassunto in italiano di Angelo Pientini (anteriore al 1588). Il riassunto di Alfonso da Spina è poi ancora ripreso ed inserito nella loro opera dai fratelli De Bry nel 1597.

c) Il Libro della Scala è soltanto citato col suo titolo arabo da Th. Bibliander nel 1543.

4. Accanto alle minori e brevi allusioni alla Visione Oltretomba di Maometto nella Spagna del Duecento, che ho sopra indicato (nel numero 1) vanno citate, perchè, al contrario di quelle, riappaiono trasmesse in successive opere, due notizie: quelle contenute nel Dialogo di 'Abdallāh ibn Salām, incluso nella Collectio Toletana (e perciò arrivato in latino nel 1141) che è la fonte di Juan de Torquemada nel 1465 ed ancora del Defensorium Fidei (anteriormente al 1473); e quelle portate nella Firenze del Trecento da Ricoldo da Montecroce (prima del 1301) e che sono state la fonte della.lettera del Papa Pio II nel 1461.

5. Alla fine del secolo XV dopo la reconquista di Granata giunge nelle letterature europee ancora dalla Spagna una nuova completa narrazione della Visione Oltretomba di Maometto: quella del faqih di Játiva: Juan Andrés. Questo dotto musulmano, convertito al 
Cristianesimo nel 1487, ed inviato a Granata dai Re Cattolici Ferdinando ed Isabella dopo la reconquista, come abbiamo veduto, inserì nella sua opera di discussione con l'Islam, una fondamentale e larga narrazione della Visione Oltretomba di Maometto, narrazione che fu tradotta dallo spagnolo ed edita in varie altre lingue europee: italiano, francese, inglese, tedesco e latino, in tutto il Cinquecento e sino al Seicento.

$\mathrm{Da}$ Juan Andrés dipendono i riassunti della Visione che ne ricavarono nei loro libri: Bernardo Perez nel 1532; Guillaume Postel nel 1543; il Guadagnoli nel 1631; ed, in parte, il Laffi nel 1683.

6. Possiamo cosi dire che, tralasciando le brevi minori fonti cui abbiamo accennato, due massime narrazioni della Visione Oltretomba di Maometto giunsero nell'Europa medievale: il Libro della Scala, nel secolo XIII; Juan Andrés nel secolo XV. Sappiamo così che la conoscenza della Visione musulmana del Paradiso e dell'Inferno era notevolmente più diffusa, nell'Europa Occidentale nel Medio Evo, di quanto si credeva sin ora.

Ed aggiungiamo che tale conoscenza derivava dalla Spagna, essendo sola eccezione il racconto di Ricoldo da Montecroce che egli portò invece da Baghdad ( $\left.{ }^{2}\right)$.

7. Ed ora in questa situazione della storia culturale del Medio Evo deve anche essere valutato il problema della Divina Commedia. Asín Palacios, quando lo pose nel 1919, trattò - a sostegno della sua ipotesi - delle comparazioni tra episodi della Commedia e passi della letteratura araba di alto livello artistico e di pensiero. Alle coincidenze cosi esposte da Asín Palacios fu opposto, come è ben noto, il fatto indubbio che Dante non conosceva l'arabo e che quelle opere della grande letteratura araba non erano state tradotte in alcuna lingua europea al tempo di Dante. Ora noi invece sappiamo che la questione va spostata in termini diversi da quelli della tesi di Miguel Asín. La Visione Oltretomba di Maometto e quindi il viaggio del Profeta dell'Islam al Paradiso ed all'Inferno era stata integralmente tradotta tra il 1260 ed il 1264 in lingue che Dante conosceva e la traduzione era opera di un Toscano: Bonaventura da Siena. Resta quindi escluso il confronto con la letteratura araba di alto livello; ma l'ipotesi di una qualche influenza della Visione

(2) Libro della Scala, pp. 346-354. Resta, per altro, ancora insoluta la questione dell'identificazione del testo di Ricoldo con il capitolo finale della Contrarietas Alpholica del codice parigino. Nationale, Lat. 3394 (loc. cit. nota 1 a p. 354). 
islamica sulla Divina Commedia va concretamente verificata sul solo testo che l'Alighieri poteva conoscere, e cioè sul Libro della Scala.

Altra volta ho già detto che, esaminando il problema in tal modo, non è assurdo formulare ipotesi sulle coincidenze tra questo o quel particolare del racconto del Libro della Scala con l'uno o l'altro particolare della Commedia. Anzi alcuni di questi incontri sembrano addirittura possibili indizi. Tuttavia, guardando più da lontano il problema, appare preferibile, considerare, al di sopra dell'esame dei particolari, la diffusa conoscenza od anche soltanto la notizia che nel mondo dell'Islam, ritenuto avversario immediato del Cristianesimo nel campo politico e religioso nella situazione storica al tempo di Dante, ma, nello stesso tempo, oggetto di ammirata assimilazione dei suoi filosofi da parte della Scolastica, si aveva come libro sacro una Visione del Paradiso e dell'Inferno. Ed appare non inverosimile che questo possa avere ispirato, insieme con altri motivi, l'Altissimo Poeta a contrapporre alla Visione islamica una Visione cristiana. E così, ripeto, non sarebbe stato nemmeno necessario che Dante avesse letto il Libro della Scala, ma soltanto che ne avesse appreso l'esistenza e quello che allora se ne riteneva il carattere sacro.

Non voglio dire così che escludo la possibile utilità dei confronti particolari, ma soltanto da quel che risulta dalla nostra ricerca sulla diffusione e la stessa redazione del Libro della Scala mi sembra opportuno insistere ancora su questa più generica possibilità entro le circostanze della cultura medievale del Trecento.

8. La questione dantesca, debbo qui ripeterlo, è quindi soltanto un episodio, anche se di particolare prestigio, di una più ampia ricerca: quanto in quel periodo era noto, nell'Europa Occidentale, della fede islamica nella vita ultra-terrena. Da quel che ho detto in questi due volumi appare chiaro con quanto interesse erano accolte in Occidente le informazioni che potevano arrivare sulla escatologia musulmana, e ciò è soltanto naturale in confronto della mentalità medievale. Riscontriamo, tuttavia, varietà di atteggiamenti. Uno è quello più semplice, e perciò di inferiore portata, ma di notevole diffusione, dei polemisti che si fermano su ciò che di materiale si interpreta delle gioie del Paradiso islamico. Altri, invece, in superiore grado e panorama culturale, pongono in antitesi proprio questa interpretazione letterale e materiale del Paradiso con le concezioni dei filosofi dell'Islam così ammirati e studiati in cuell'epoca; e pertanto nasce la tradizione che, da Guillaume d'Auvergne, agli inizi 
del Duecento $\left({ }^{3}\right)$, giungerà almeno sino a Filippo Guadagnoli nel Seicento $\left({ }^{4}\right)$, rappresenterà i filosofi Avicenna ed Averroes contrari a quella materialità dell'Oltretomba e sostenitori di una interpretazione allegorica del Corano. Ed in questa idea gli Occidentali erano forse aiutati dall'imbarazzo col quale i filosofi musulmani avevano cercato di conciliare i loro sistemi con la teologia ortodossa.

9. In questi ambienti di maggior cultura filosofica doveva poi esser composta, come abbiamo visto, alla fine del secolo XII od ai primi del Duecento - e forse alla corte dei Re Normanni di Sicilia - un Itinerario dell'Anima (in latino) nei regni oltretomba (Paradiso ed Inferno). L'Itinerario era impersonale e puramente filosofico e, per quanto concerne l'Islam, in stretta relazione con la filosofia di Avicenna e senza alcuna relazione, invece, con la tradizione del viaggio di Maometto nell'Oltretomba. L'Anima sale per dieci gradi paradisiaci e scende per dieci miseriae nell'abisso infernale. Nessun nome, nessun personaggio, niente al di fuori delle concezioni filosofiche $\left.{ }^{5}\right)$. Soltanto la forma esteriore di "Itinerario" richiama quella della tradizione comune.

Ed ancora nella metafisica di Al-Gazzāli, tradotta in latino da Giovanni di Siviglia, si poneva come pena dell'Inferno la estrema esagerazione delle passioni, dei desideri e dei vizi che $i$ singoli dannati avevano avuto in vita, esagerazione passionale che era accompagnata dalla sensazione che nulla più poteva essere soddisfatto di quei desideri, passioni e vizi dopo la estinzione della vita corporea. In questa contrapposizione era cosi il cruciatus ineffabilis (6). Tale proposizione veniva forse a coincidere con quella della immaterialità delle pene dell'Inferno che Etienne Tempier, Vescovo di Parigi, aveva condannato nel 1277. Ma ancora qui siamo rigorosamente nel campo filosofico, anche se già notai altrove come - senza necessariamente supporre una relazione di fonte - il concetto di alGazzāli richiama le pene di Capaneo e di Francesca da Rimini nella Commedia.

Comunque l'assimilazione della filosofia musulmana in Occidente non mancò di suscitare reazioni per contenere entro l'ortodossia gli entusiasmi che quelle dottrine avevano suscitato nelle scuole. Per-

(s) Libro della Scala, pp. 402-412.

(4) Cfr. qui sopra pp. 236-237.

(5) Libro della Scala, pp. 519-522.

( ${ }^{\circ}$ Libro della Scala, pp. 516-517. 
tanto è tipico l'opuscolo De erroribus ed il conseguente scritto, nel complesso meno tagliente, delle istruzioni che nel 1358 Nicola Aymeric, Grande Inquisitore di Aragona indirizzava ai suoi dipendenti ( 7 ).

Tra le generiche ragioni popolarmente diffuse sull'Islam ed il "Paradiso di Maometto" e le elevate elaborazioni dei filosofi trova, dunque, posto - ad un livello medio - il Libro della Scala e perciò le conoscenze che ne derivano negli ambienti culturali del Trecento ed oltre, come abbiamo visto. E, se oltre alla storia dei rapporti e delle discussioni tra l'Occidente Cristiano e l'Islam in quel periodo del Medio Evo, vogliamo guardare alla letteratura ed all'arte, riconosceremo ora che ispirazioni agli Occidentali potevano arrivare dall'uno o dall'altro di questi tre livelli di conoscenza dell'Islam.

10. In un altro particolare settore di tale conoscenza ancora appaiono ad un tempo questi stessi tre elementi, ed ancora - però qualcosa di nuovo. Alludo alle tradizioni sui prodigi di Maometto arrivate nelle letterature dell'Occidente. Le discussioni, come ho già detto, citavano così spesso e con tanto vigore queste tradizioni che la conoscenza delle loro possibili svariate origini ha importanza notevole per ricostruire proprio la storia dei rapporti con l'Islam su tali temi. Ora dall'esame che ne abbiamo fatto sopra nella seconda parte di questo volume risulta che appunto tali tradizioni hanno queste fonti:

a) racconti di pietà popolare del mondo islamico, che gli Occidentali facilmente, se pure erroneamente, hanno ritenuto parte essenziale delle credenze dei Musulmani

b) racconti popolari immaginati in Occidente - alcuni adattando antiche favole, come quello della calamita sulla tomba; altri adattando leggende medievali da epopea, come quelli su Maometto e l'Imperatore Erachio, o motivi locali spagnoli, come quelli su Maometto e S. Isidoro di Siviglia, e racconti formati con intenzione non amichevole per l'Islam, negli ambienti popolari cristiani orientali;

c) racconti che ampliano passi del Corano i quali costituiscono il punto di partenza per più diffuse precisazioni, come quello della luna spaccata. Ed a tale categoria, se non avesse avuto un tanto maggior sviluppo letterario, apparterrebbe anche la stessa Visione

(7) Cfr. qui sopra pp. 301-312. 
Oltretomba di Maometto, che parte, infatti, dalla sura XVII del Corano.

d) infine i racconti di prodigi che hanno assunto nelle letterature musulmane un valore ed un significato non più letterale, ma di simbolo mistico, come la purificazione del cuore di Maometto e la Luce del Profeta.

Il dato nuovo è appunto la raccolta di tradizioni popolari di Musulmani e di Cristiani di Palestina, raccolta fatta dai pellegrini europei in Terra Santa e da essi inclusa nei loro Itinerari. Ma questo non appare che nel secolo XVII; e per i secoli anteriori resta assoIuta fonte quanto era filtrato in Occidente dalla Spagna musulmana.

Così, mentre nella filosofia e nelle scienze i contatti dell'Europa Occidentale col mondo musulmano avevano tanta parte nel progresso della cultura medievale, anche nel campo più delicato - e più particolarmente delicato - delle tradizioni religiose islamiche i contatti furono assai maggiori di quanto si fosse sin ora supposto. Ai nomi grandissimi dei santi dottori Alberto Magno e Tommaso d'Aquino e dei filosofi Avicenna, Averroes ed Algazel, a quelli di al-Battānī, al-Huwārizmī, al-Birūnì e del Sacrobosco, Michele Scoto, Leonardo Fibonacci e di altri sono legati quei successi massimi che da Cordova per tutti i Paesi Arabi e sino all'Asia Centrale, da un lato, e da Toledo per la Catalogna, Parigi, Oxford, Bologna e Padova sino alla Sicilia hanno trasformato il pensiero e le attività umane. Contemporaneamente nella vita popolare giornaliera e nelle credenze minute, racconti e nozioni, edificanti o polemiche, passavano dall'uno all'altro campo. A metà del cammino e più in alto di questo livello minimo la dotta curiosità di Alfonso il Savio ha dato il Libro della Scala, e con il loro Sovrano abbiamo i nomi di Abraham Alfaquím (8), e Bonaventura da Siena; come alla fine del Medio Evo l'intervento dei Re Ferdinando ed Isabella ci darà l'opera di Juan Andrés.

Aggiungeremo a questa lista il nome dell'Altissimo Poeta? L'interrogazione è ora circoscritta nei suoi limiti. Dante poeta viveva nell'ambiente culturale del suo tempo quando la situazione

(8) Alle notizie sulla persona di Abraham Alfaquím da me riunite nel Libro della Scala, pp. 17-22 conviene ora aggiungere la notizia data da Davide Romano in Atti del Convegno Internazionale: Oriente ed Occidentc. Filosofia e Scienze, Roma, Accademia Lincei 1971, p. 69J nota 93. 
tra Oriente musulmano ed Occidente era quella dei contatti, dal massimo all'infimo grado, che qui abbiamo delineato. Alla tradizione filosofica Dante nella Commedia (e nel Convito) si riferisce, anche esplicitamente, ma di solito non per conoscenza diretta delle traduzioni dei filosofi arabi, bensi attraverso le loro citazioni negli scritti di S. Alberto Magno e S. Tommaso d'Aquino. La tradizione popolare gli è nota ed il $28^{\circ}$ Canto dell' Inferno ne è una prova. $\mathrm{Ha}$ conosciuto direttamente anche il Libro della Scala nella traduzione del suo conterraneo Bonaventura da Siena? oppure la Visione musulmana dell'Oltretomba, ed indirettamente, quindi, la Scala, ha potuto - insieme ad altri motivi - spingere 1'Alighieri a contrapporre una Visione cristiana della vita ultraterrena? Abbiamo qui presentato in questi due volumi i molteplici dati dai quali le ipotesi possano prendere il volo; e mi auguro di poter dire della mia fatica con i versi del Paradiso:

Pensa, lettor, se quel che qui si inizia non procedesse, come tu avresti di più sapere angosciosa carizia.

Pur tuttavia non tralasciamo che le maggiori notizie sulla diffusione del Libro della Scala, nello spazio e nel tempo, rendano meno assurdo che l'Alighieri, direttamente od indirettamente, ne abbia avuto notizia.

Ma a noi preme più generalmente il grande fenomeno storico della fioritura degli scambi intellettuali tra il mondo islamico e quello dell'Europa d'allora ed il rinnovamento del pensiero occidentale che ne fu la conseguenza, segnando profondamente la civiltà umana in quell'ambiènte dell'unità culturale dei Paesi Mediterranei che è stata, ogni volta che fu realizzata, madre di progresso umano. 